\title{
A formula to estimate water loss in a malnourished patient with ascites: One inch equals one kilogram
}

\author{
Sevan V Stepanian ${ }^{1}$, John A. Tayek*2 \\ ${ }^{1}$ David Geffen School of Medicine, Torrance, CA, USA \\ ${ }^{2}$ David Geffen School of Medicine, Harbor-UCLA Medical Center, Torrance, CA, USA
}

Received: May 31, 2016

DOI: $10.5430 /$ crim.v3n4p7
Accepted: July 14, 2016

Online Published: August 7, 2016

\begin{abstract}
Severe ascites accounts for a large percentage of body water, making measurements of lean body mass (LBM) inaccurate. There are no bed-side methods to determine the contribution of ascites to LBM. Using the formula for a sphear, a patients volume of ascites was obtained by the standard equation (Volume $=4 / 3 \times \mathrm{Pi} \times[\text { radius }]^{3}$ ). A 54-year-old malnourished Hispanic female with Child's Class C liver cirrhosis presented to the ER with 2-weeks of increasing abdominal pain, girth, fever, chills and decreased nutritional intake. The admission abdominal girth measured $106 \mathrm{~cm}$ at the umbilicus. The radius of the sphere was obtained measuring the abdominal girth divided by two times Pia times radius (radius $=106 \mathrm{~cm} /[2 \times 3.14]=16.9 \mathrm{~cm}$ ). Using the above calculated radius of $16.9 \mathrm{~cm}$, Volume $=4 / 3 \times 3.14 \times 16.9 \mathrm{~cm}^{3}=20,208 \mathrm{cc}=$ approximate volume of the abdomen at presentation. Using the same formulas, abdominal volume was $8,275 \mathrm{~cm}$ cubed seven days after discharge from hospital. The difference in abdominal volume from admission to follow up is $20,208 \mathrm{cc}-8,375 \mathrm{cc}=11,833 \mathrm{cc}$, assuming a specific gravity of 1.0, 11.8-liters of volume or kg of weight. The amount of ascitic abdominal fluid lost was confirmed by comparing change in volume to change in body weights (66.0 kg-55.0 kg, admission and follow-up weights, respectively). The $11.0 \mathrm{~kg}$ weight loss was similar to the estimated change in abdominal body water change of $11.8 \mathrm{~kg}$. Conclusion: The simple measurement of waist circumference may provide an estimate of ascites volume and changes in volume should be similar to changes in weight. For every $2.4 \mathrm{~cm}$ ( 1 inch) that the waist circumference is reduced, body weight (and ascites) should be reduced by 1-kilogram.
\end{abstract}

Key Words: Cirrhosis, Branched-chain amino acids, Anthropodometry, Anthropometrics, Third spacing, Tissue edema, Ascites, Spontaneous bacterial pertionitis

\section{INTRODUCTION}

Earlier work with 23 cirrhotic patients with cirrhosis demonstrated that $46 \%$ of measured weight loss was due to a reduction in measured ascites volume using methylene blue dilution to measure the volume. ${ }^{[1]}$ This is the first report using the formula of a sphere to estimate ascites volume and relate this to change in weight loss after resolution of the ascites volume.

\section{CASE REPORT}

A 54-year-old malnourished Hispanic female with Child's Class $\mathrm{C}$ liver cirrhosis presented to the emergency department with 2-weeks of worsening abdominal pain, increasing abdominal girth, fever, chills and decreased oral intake. Admission weight was $66.0 \mathrm{~kg}$. The physical exam revealed scleral icterus, a soft but distended abdomen measuring 106 $\mathrm{cm}$ in circumference, tender to palpation in all four quadrants,

\footnotetext{
* Correspondence: John A. Tayek, MD, Professor; Email: jtayek@dhs.lacounty.gov; Address: David Geffen School of Medicine, Harbor-UCLA Medical Center, 1000 W. Carson Street, Torrance, CA 90509, USA.
} 
and extremity examination revealed $2+$ edema. Admission laboratory values revealed an albumin of $1.5 \mathrm{mg} / \mathrm{dl}$, INR of 1.6, BUN 50. Paracentesis on admission revealed ascitic fluid containing 2,100 white blood cells. She was subsequently treated for spontaneous bacterial peritonitis (SBP) with $1 \mathrm{~g}$ IV Ceftazidime. The patient continued to have fevers despite ceftazidime treatment and a second paracentesis evidenced continuous infection with neutrophils (74\%) so Ceftazidime was replaced with Piperacillin-tazobactam (Zosyn). Throughout the course of her stay the patient was placed on a high branched chain amino acids (BCAA) supplement called NutraHep ( 1 can twice a day) and partial parenteral nutrition consisting of $50 \%$ BCAA. The patient's symptoms improved with continued antibiotic and nutritional therapy and she was subsequently discharged home with medications for SBP prophylaxis and portal hypertension as well as nutritional therapy NutraHep. The patient's albumin level at discharge was $2.2 \mathrm{mg} / \mathrm{dl}$ and her abdominal circumference measured one week after her discharge date was noted to be $79 \mathrm{~cm}$ and her weight was $55 \mathrm{~kg}$.

The above case provides an opportunity to derive a simple formula to estimate ascitic fluid volume semicolon, which may be used as a potential objective marker to monitor response to therapy. Using the formula to determine the volume of a sphere, we can estimate the volume of the abdominal contents given the patient's abdominal circumference. In the above case admission abdominal girth measured at 106 $\mathrm{cm}$. The radius of the sphere can be obtained by dividing the circumference by $2 \times \mathrm{Pi}$. (radius $=106 \mathrm{~cm} /[2 \times 3.14]$ $=16.9 \mathrm{~cm}$ ). The approximate volume of the abdomen can be estimated from the formula to determine the volume of a sphere, $4 / 3 \times \mathrm{Pi} \times(\text { radius })^{3}$. Using the above calculated radius of $16.9 \mathrm{~cm}$, Volume $=4 / 3 \times 3.14 \times 16.9 \mathrm{~cm}^{3}=20,208$ $\mathrm{cc}=$ approximate volume of the abdomen at presentation.

Using the same formulas, we can estimate the patient's abdominal volume at her 1-week outpatient clinic visit using her abdominal circumference, which was measured at 79 $\mathrm{cm}$. radius $=79 \mathrm{~cm} /(2 \times 3.14)=12.6 \mathrm{~cm} ;$ and Volume $=$ $4 / 3 \times 3.14 \times 12.63=8,375 \mathrm{cc}$ approximate volume of the abdomen at follow up. The difference in abdominal volume from admission to follow up is $20,208 \mathrm{cc}-8,375 \mathrm{cc}=11,833$ cc or, assuming a specific gravity of 1.0, 11.8 liters of volume and $11.8 \mathrm{~kg}$ of weight.

This approximation of the amount of ascitic fluid lost can be confirmed by comparing it to the difference of the admission and follow up visit weights. This difference in weight from the two visits is $11.0 \mathrm{~kg}(66.0 \mathrm{~kg}-55.0 \mathrm{~kg})$ which is very similar to the amount of weight loss estimated by using the difference in ascitic fluid volume from admission to the follow up visit (11.8 $\mathrm{kg}$ as calculated above).

The extent of exactly what percentage of the additional $11,833 \mathrm{cc}$ of abdominal volume was due to ascitic fluid was estimated by applying the Vitrea Imaging Software to an abdominal CT scan obtained at admission. Using this software semicolon allowed us to reconstruct a three-dimensional representation of the ascitic fluid in the abdomen and then calculate the volume within it. We calculated the ascitic fluid volume to be $2,941 \mathrm{cc}$, which is $25 \%$ of the additional volume of the abdomen $(11,833 \mathrm{cc})$. The remaining $75 \%$ of the volume may be attributed to edema semicolon of various tissues within the abdomen including the bowel and liver. A likely smaller amount of weight loss would be due to the loss of weight from the lower extremities. Earlier work with 23 patients with cirrhosis demonstrated that $46 \%$ of measured weight loss was due to a reduction in measured ascites volume using methylene blue dilution to measure the volume. ${ }^{[1]}$ Using CT with volume ascites measurements documented that the water volume loss represented $25 \%$ of the total weight loss. Most of the loss was due to reduction in abdominal tissue, liver and other organ edema that resolved with resolution of the ascites and abdominal tissue edema.

The differences in volume and weight in this patient, pre and post treatment, compared to pre and post treatment abdominal circumference allows us to derive a simple formula to estimate excess abdominal fluid volume in patients with ascites. The $27 \mathrm{~cm}$, or 10.6 inch, reduction of abdominal circumference measured in our patient correlates with 11.0 $\mathrm{kg}$ of weight reduction. By reducing the above terms we can then show that one-inch of abdominal girth loosely correlates with $1 \mathrm{~kg}$ of weight loss or $1 \mathrm{~L}$ of abdominal volume.

Patients with ascites have an increased energy expenditure, which falls after removal of the ascites. ${ }^{[2]}$ Furthermore, increased calorie intakes have been associated with a prolonged 6-month survival in patients with severe ascites. ${ }^{[3]}$ The decision to initiate a high calorie diet alone with BCAA supplemented nutritional therapy was made with consideration of the results from a year long, large multicenter, randomized controlled trial in 2003 semicolon comparing BCAA enriched nutritional therapy to lactoalbumin or maltodextrin in patient with liver cirrhosis. Patients who received BCAA therapy showed significant reduction in disease progression. ${ }^{[4]}$

Recent study using ultrasound demonstrated that $1 \mathrm{~cm}$ of ultrasound measurement in a pocket of ascites fluid relates to a 1 liter amount of fluid. ${ }^{[5]}$ Using $1 \mathrm{~cm}$ as the measurement of diameter for volume measurement would extrapolate in the change in abdominal volume of 1.8 liters in your patient. Both the pocket measurement and the abdominal waist mea- 
surement may be helpful tools to estimate ascites volume for the clinician.

\section{Conclusion}

The antibiotic treatment and the aggressive nutritional support resulted in complete resolution of this patient's ascites within a 16-day period. The simple measurement of waist circumference may provide an estimate of ascites volume and changes in volume should be similar to changes in weight. For every $2.4 \mathrm{~cm}$ (1 inch) that the waist circumference is reduced, body weight should be reduced by 1-kilogram. Ascites fluid accounts for only $25 \%$ of the observed weight loss after resolution of severe ascites and SPB. The majority of the weight and fluid loss is due to loss of fluid in edematous organs and subcutaneous tissue. Further work is needed to validate the easy use of abdominal waist circumference in estimating ascites volume and the response with therapy.

\section{Consent}

Written informed consent was obtained from the patient for publication of this case report.

\section{Authors contributions}

JT obtained data and finalized the draft report and validated all details of the case report.

\section{ACKNOWLedgements}

Thank you to Cindy L. Tayek M.S.N. for critical review and editing of manuscript.

\section{CONFLICTS OF INTEREST Disclosure}

The authors have declared no conflicts of interest.

\section{REFERENCES}

[1] Milani A, Ciammella AM, Rossi L. Ascites free-water dynamics in de-compensated cirrhosis: effect of acute and chronic furosemide treatment. Eur J Gastroenterol Hepatology. 1995; 7(8): 763-777. PMid:7496866

[2] Dolz C, Raurich JM, Ibanez J, et al. Ascites increases the resting energy expenditure in liver cirrhosis. Gastroenterology. 1991; 100(3): 734-744.

[3] Marchesini G, Bianchi G, Merli M, et al. Abbiati R for the Italian BCAA Study Group. Nutritional supplementation with branchedchain amino acids $\mathrm{n}$ advanced cirrhosis: A double blind randomized trial. Gastroenterology. 2003; 124: 1792-1801. http://dx.doi.o $\mathrm{rg} / 10.1016 / \mathrm{S} 0016-5085$ (03) 00323-8

[4] Mendenhall CL, Moritz TE, Roselle GA, et al. Veterans Affairs Cooperative Study Group 275. A study of oral nutritional support with oxandrolone in malnourished patients with alcoholic hepatitis: Results of a Department of Veterans Affairs Cooperative Study. Hepatology. 1993; 17(4): 564-76. PMid:8477961 http: //dx.doi.org/10.1002/hep.1840170407

[5] Irshad A, Ackerman SJ, Anis M, et al. Can the smallest depth of ascitic fluid on sonograms predict the amount of drainable fluid? J Clin US. 2009; 37(8): 440-44. PMiD:19618437 http://dx.doi.o $\mathrm{rg} / 10.1002 / \mathrm{jcu} .20616$ 